\title{
Validação Experimental de Modelos de Leitos Adsorvedores com Zeólitas 5A
}

\section{(Experimental Validation of Models of 5A Zeolites Adsorbing Beds)}

\author{
Vanderley de Vasconcelos, Mônica Schvartzman, Wagner Reis da Costa Campos \\ Centro de Desenvolvimento da Tecnologia Nuclear - CDTN/CNEN \\ Caixa Postal 941, CEP 30.123-970, Belo Horizonte, MG \\ e-mail:vasconv@urano.cdtn.br
}

Wander L. Vasconcelos

Universidade Federal de Minas Gerais - Dep. Eng. Metalúrgica e de Materiais

Rua Espírito Santo 35, CEP 30.160-030, Belo Horizonte, MG

e-mail:wlv@urano.cdtn.br

\section{Resumo}

A caracterização de meios porosos é muito importante em áreas científicas e tecnológicas envolvendo materiais e processos químicos. Processos de transferência de calor e massa em meios porosos dependem, além de suas propriedades métricas, das propriedades topológicas e topográficas. No entanto, essas propriedades são de difícil acesso experimental, razão pela qual torna-se necessária, algumas vezes, a utilização de técnicas de simulação para auxiliar nas suas determinações. Este trabalho apresenta os resultados de simulação de leitos adsorvedores utilizando zeólitas $5 \mathrm{~A}$ comerciais. Os modelos de compactação de partículas esféricas dos leitos adsorvedores são ajustados para que suas propriedades métricas, como fração volumétrica e área superficial de poros, se aproximem daquelas determinadas para os leitos reais. A partir dos modelos ajustados, os parâmetros topográficos e topológicos, como a distribuição radial da porosidade e a conectividade de poros, são estimados e comparados com valores obtidos para os leitos reais através de métodos indiretos.

\section{INTRODUÇÃO}

Processos de separação por adsorção têm sido utilizados na indústria em substituição a técnicas convencionais. Nos leitos adsorvedores, o grau de compactação das partículas, a distribuição radial de porosidade e a conectividade dos poros formados entre as partículas adsorventes influenciam diretamente os processos de transferência de calor e massa [1]. Os efeitos das bordas ("wall effects") são fatores importantes na análise e projeto de leitos adsorvedores. A variação radial da porosidade influencia significativamente os perfis de temperatura e velocidade nos leitos. Os modelos utilizados para prever a distribuição radial de porosidade

\section{Abstract}

The characterization of porous bed is of crucial importance in many scientific and technological areas related to materials and chemical processes. Mass and heat transfer in a porous media depend on metric, topological and topographical properties. Because the difficulties of experimental access to topological and topographical properties sometimes it is practical the use of computer simulation techniques to evaluate those parameters. This paper presents the simulation results of adsorbing beds using commercial 5A zeolites. The sphere packing models applied to adsorbing beds are adjusted through the comparison of metric properties, such as volumetric fraction and specific area of pores, evaluated for simulated and real beds. Starting from adjusted models, the topological and topographical properties, like radial distribution of porosity and pore connectivity are estimated and compared with those parameters obtained for real beds through the use of indirect methods.

são normalmente muito simplificados e não prevêem oscilações amortecidas da porosidade radial, nem partículas de diferentes tamanhos [2]. Por isso muitas vezes os valores previstos por esses modelos não estão de acordo com os valores experimentais.

As determinações do número de coordenação de partículas e da conectividade de poros requerem a realização de seções seriadas e o acompanhamento das variações das propriedades topológicas de cada elemento de uma seção para outra [3]. Isso demanda muito tempo e esforço, tornando difícil a previsão de parâmetros importantes para a separação de gases via leitos adsorvedores, como por exemplo a permeabilidade.

O propósito deste trabalho é desenvolver uma modelagem 
geométrica para o leito adsorvedor com partículas de zeólitas 5A, através da implementação de modelos de compactação de partículas em um ambiente de computação gráfica e modelagem de sólidos (AutoCAD). Esses modelos são utilizados, após as respectivas etapas de validação, como auxílio na determinação dos parâmetros de difícil acesso experimental.

\section{CARACTERÍSTICAS ESPECÍFICAS DE ZEÓLITAS CO- MERCIAIS 5A E DE LEITOS ADSORVEDORES}

A medida da distribuição de tamanho de partículas de zeólitas comerciais $5 \mathrm{~A}$, realizadas através de separação granulométrica por peneiramento, mostra claramente que a distribuição é bimodal, com a presença de partículas com diâmetro médio de 2,38 $\mathrm{mm}$ e partículas pequenas com diâmetro médio de $1,68 \mathrm{~mm}$, numa proporção de $20 \mathrm{w} \%$ e $80 \mathrm{w} \%$ respectivamente. A forma das partículas se aproxima bastante da esférica, apresentando, no entanto, algumas partículas quebradas, conforme pode ser verificado na Figura 1. Essas partículas serão utilizadas em uma unidade piloto de separação de gases, projetada e montada no CDTN. A unidade é composta de um leito adsorvedor de aço inoxidável, com diâmetro de $25 \mathrm{~mm}$ e altura de $400 \mathrm{~mm}$, contendo zeólitas tipo 5A, válvulas solenóides controladas por temporizadores e dispositivos de medição de pressão, temperatura e vazão.

As densidades dos leitos adsorvedores, medidas através do método gravimétrico foram: $0,86 \mathrm{~g} / \mathrm{cm}^{3}$, para o leito preenchido com esferas grandes e pequenas na proporção de 20 w $/$ e 80 w/, respectivamente; $0,87 \mathrm{~g} / \mathrm{cm}^{3}$, para o leito preenchido somente com esferas grandes; $0,90 \mathrm{~g} / \mathrm{cm}^{3}$, para o leito preenchido somente com esferas pequenas.

É um fato conhecido que a distribuição radial de porosidade em leitos com esferas de mesmo tamanho, distribuídas aleatoriamente, é afetada pela proximidade com as paredes dos recipientes. A primeira camada em contato com a parede tende a ser bem ordenada, com a maioria das esferas tocando a parede. À medida que se caminha na direção do centro do leito, as camadas tendem a ser cada vez mais desordenadas até que, longe da parede é obtida uma

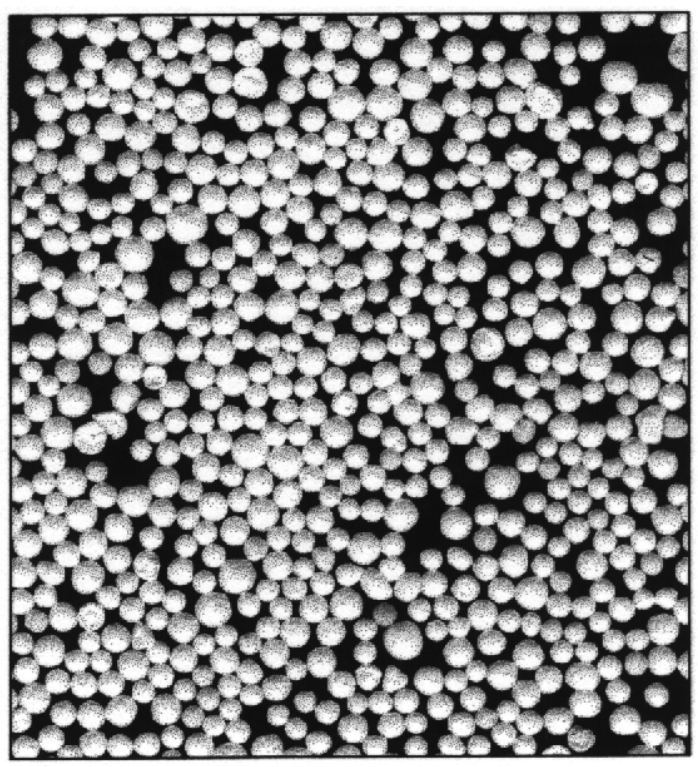

Figura 1: Partículas de zeólitas comerciais 5 A fabricadas pela Bayer. configuração completamente aleatória. Pesquisas realizadas neste campo e publicadas desde 1958 têm demonstrado claramente que essas distribuições radiais de porosidade oscilam de forma amortecida a partir da porosidade do leito, na direção do seu centro [2]. Um parâmetro que influencia bastante a distribuição radial de porosidade é a razão de aspecto, definido como a razão entre o diâmetro do leito e o diâmetro das esferas. Para valores de razão de aspecto muito grandes (acima de 20) as oscilações têm um alcance de quatro a cinco diâmetros das esferas a partir da parede do leito. Leitos com razões de aspecto menores que 10 não apresentam quaisquer regiões com porosidades constantes.

Baseadas nessas observações experimentais, têm sido desenvolvidos alguns modelos empíricos relacionando a porosidade radial com a razão de aspecto e a distância da parede $[2,4]$. Esses modelos têm sido utilizados em modelos analíticos de transporte para simular leitos empacotados de partículas esféricas em recipientes cilíndricos. Seus resultados serão úteis como métodos indiretos para validar os modelos de simulação empregados.

\section{SIMULAÇÃO DO LEITO DE ZEÓLITAS}

Para a simulação do leito de zeólitas foi implementado um algoritmo computacional no qual partículas esféricas caem a partir de uma posição aleatória do topo do leito cilíndrico. Durante a sua queda, cada partícula alcança uma posição estável quando ocorrer uma das seguintes situações: atingir o fundo do leito; atingir duas partículas e a parede do leito; e atingir três outras partículas simultaneamente. Em alguns casos particulares nas duas últimas situações apresentadas as partículas podem ainda não estar em posições estáveis e devem ainda continuar o processo de queda [5]. Foram também implementados algoritmos que permitem a movimentação das esferas no fundo do leito cilíndrico, ao serem atingidas por alguma esfera em processo de queda. Esses critérios de estabilidade estão ilustrados na Figura 2 e dependem basicamente da semireta vertical $m$, que passa pelo centro da esfera em movimento $M$. Se $m$ cruzar o interior do triângulo formado pelo centro das três esferas $(A, B e C)$ ou duas esferas e o ponto $P$ de contato com a parede, $M$ será estável. Caso contrário, $M$ será instável. Um leito

Tabela I: $V^{p}{ }_{v}$ em leito real e leito simulado com (a) algoritmo que não considera movimento das partículas no fundo do leito e (b) algoritmo que considera movimento das partículas no fundo do leito.

\begin{tabular}{llll}
\hline $\begin{array}{l}\text { Tamanho de } \\
\text { Partículas }\end{array}$ & $\begin{array}{l}\text { Leito } \\
\text { Simulado }\end{array}$ & $\begin{array}{l}\text { Leito } \\
\text { Real }\end{array}$ \\
\hline & $\mathrm{V}_{\mathrm{v}}^{\mathrm{p}}(\mathrm{a})$ & $\mathrm{V}_{\mathrm{v}}^{\mathrm{p}}(\mathrm{b})$ & $\begin{array}{c}\mathrm{V}_{\mathrm{v}}^{\mathrm{p}} \text { (método } \\
\text { gravimétrico) } \\
\end{array}$ \\
& $\%$ & $\%$ & $\%$ \\
\hline Grandes & 47,3 & 43,0 & 39,2 \\
\hline Pequenas & 44,9 & 40,9 & 37,1 \\
\hline $20 \mathrm{w} / 80 \mathrm{w} / \mathrm{o}$ & 45,4 & 41,2 & 37,7 \\
\hline
\end{tabular}




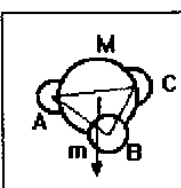

[a]

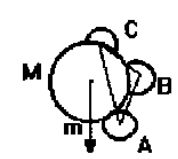

[b]

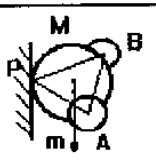

(c)

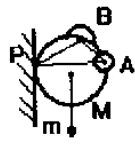

[d]
Figura 2: Critérios de estabilidade para uma esfera em contato com outras três ou com duas esferas e a parede (a e c estáveis e b e d instáveis).

adsorvedor tridimencional simulado utilizando esses critérios, para partículas esféricas de mesmo tamanho, é mostrado na figura 3.

Foi feita uma comparação entre as frações volumétricas de poros $\left(\mathrm{V}^{p}{ }_{v}\right)$ medidas nos leitos simulados utilizando os algoritmos descritos e aquelas medidas nos leitos reais através de métodos gravimétricos (Tabela 1). Os valores obtidos para $\mathrm{V}^{p}{ }_{v} \operatorname{com} \mathrm{o}$ algoritmo de simulação que considera movimento das partículas no fundo do leito se aproximam mais dos valores medidos para os leitos reais, o que faz com que este algoritmo de simulação seja o mais adequado para representar o leito de zeólitas em análise.

Para o leito simulado com dois tamanhos de partículas foi medida a distribuição radial de porosidade e os resultados são mostrados na Figura 4 . Nota-se a presença das oscilações amortecidas, previstas pelos modelos que levam em conta um único tamanho de partículas, no entanto, com características diferenciadas com relação a amplitudes e períodos, provocadas pela presença dos dois tamanhos.[6]

\section{SEÇÕES SERIADAS NO LEITO REAL}

A realização das seções seriadas em amostras dos leitos reais, além de possibilitar a validação de valores estimados da distribuição radial da porosidade e conectividade, possibilita uma avaliação direta de métodos estereométricos como o método de Saltykov. Isto é possível porque a distribuição de tamanho de partículas obtida a partir de uma seção pode ser comparada com a distribuição de tamanho de partículas estimada através do uso de seções seriadas.

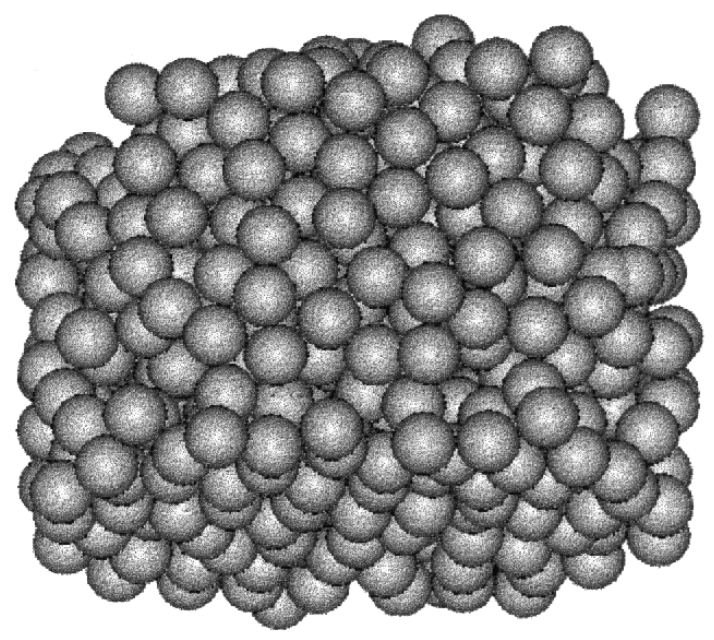

Figura 3: Leito adsorvedor tridimensional simulado num ambiente de computação gráfica.

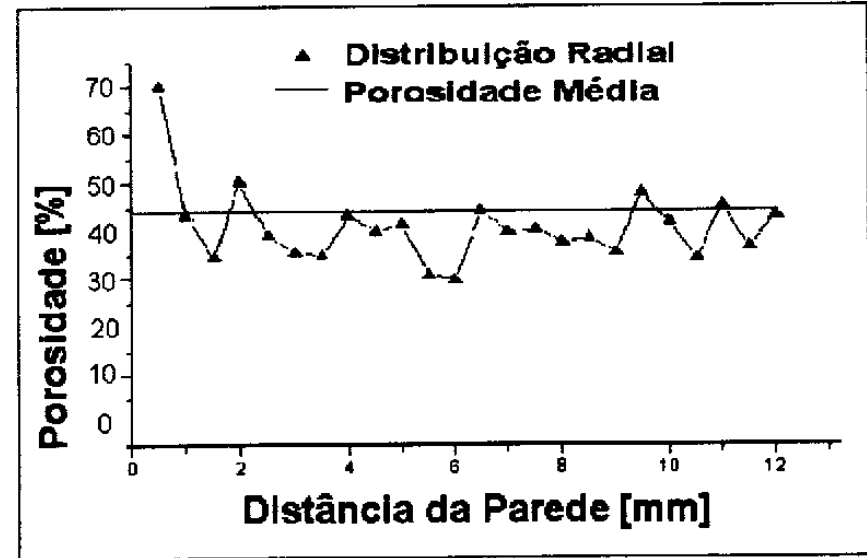

Figura 4: Distribuição radial da porosidade estimada para o leito simulado com esferas grandes e pequenas.

Foram realizadas 12 seções seriadas em uma amostra do leito contendo partículas grandes de zeólita $5 \mathrm{~A}$ e medida, para cada uma, a espessura do leito com um micrômetro. Cada uma das seções foi diretamente digitalizada em um scanner com uma resolução de $600 \mathrm{dpi}$ e impressa em transparência para que cada esfera de zeólita pudesse ser acompanhada em seções consecutivas, com a finalidade de determinar o seu diâmetro e as coordenadas do seu centro. O diâmetro de cada esfera foi estimado a partir dos diâmetros de duas de suas seções em planos de corte consecutivos.

Com a finalidade de determinar o número de coordenação das esferas no trecho seccionado da amostra do leito foram posicionadas, no ambiente de computação gráfica com modelagem de sólidos, 254 esferas com os diâmetros e coordenadas determinadas pelas seções seriadas. A comparação entre a distribuição dos números de coordenação obtidos para o leito simulado, com esferas de mesmo tamanho, com os valores obtidos para a amostra do leito real é mostrada na Figura 5.

O número de coordenação médio maior do leito real $(6,2$ para o leito real e 5,7 para o leito simulado) pode ser explicado pela menor fração volumétrica deste em relação ao leito simulado $(39,2 \%$ em comparação com $43,0 \%$ ) e pelo fato de terem sido utilizadas, para a simulação, esferas de mesmo tamanho e não a distribuição de tamanhos "real" determinada nas zeólitas 5A utilizadas. No entanto,

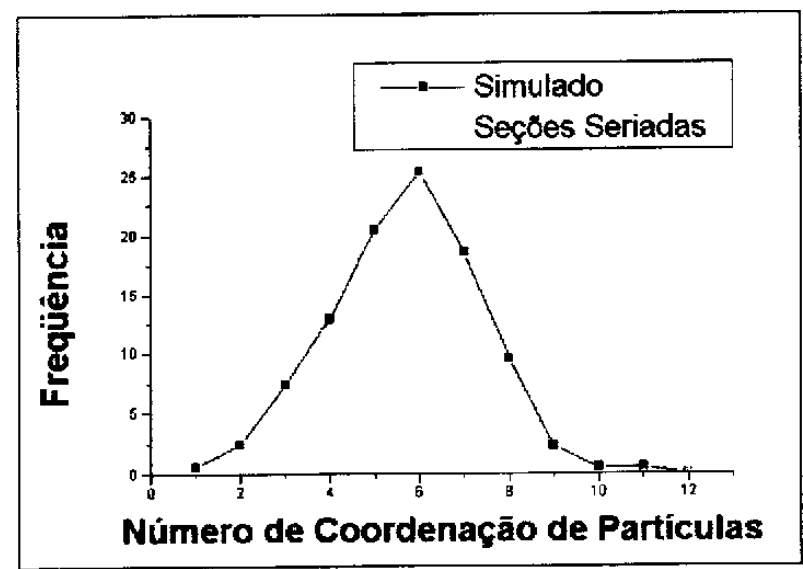

Figura 5: Comparação entre os números de coordenação de partículas no leito adsorvedor simulado e no leito real. 
o erro cometido na estimativa da conectividade do leito utilizando os resultados de simulação é inferior a $9 \%$, o que os torna adequados para a maioria das aplicações, principalmente se for considerado que este é um parâmetro de difícil acesso experimental.

O achatamento da curva de distribuição de números de coordenação, obtida pela técnica de seções seriadas, nas suas extremidades, se deve ao fato de existir uma faixa de diâmetros de esferas, ao invés de um único tamanho, conforme considerado na simulação. $\mathrm{O}$ efeito de se introduzir partículas maiores em um conjunto de partículas menores de mesmo tamanho, por exemplo, é aumentar a freqüência dos números de coordenação maiores, correspondentes às partículas maiores introduzidas que se cercarão de um maior número de partículas menores. Isto faz com que a curva seja achatada no seu lado direito. Além disso, as partículas menores em contato com as partículas maiores tenderão a ter seus números de coordenação reduzidos, fazendo com que a curva seja achatada também para a esquerda. A variação do diâmetro, por influir na razão de aspecto, também influencia diferentemente os números de coordenação das partículas próximas às paredes do leito real, em relação ao leito simulado que considera um único tamanho de partículas.

\section{CONCLUSÕES}

Pelos resultados obtidos de frações volumétricas de poros e números de coordenação médios de partículas, para os leitos simulados e os leitos reais, foram verificadas diferenças entre os resul- tados obtidos para estas duas variáveis abaixo de $10 \%$. Isto indica que os algoritmos de simulação geométrica computacional implementados poderão ser usados para a previsão de propriedades e projeto de leitos adsorvedores. As distribuições radiais de porosidades obtidas pelas simulações estão de acordo com aquelas previstas pelos modelos analíticos e com os resultados obtidos a partir das seções seriadas. Pode então ser estabelecido um procedimento para determinar a distribuição radial da porosidade e a conectividade para leitos de zeólitas 5A, tomando como base os algoritmos de simulação implementados.

\section{AGRADECIMENTOS}

Os autores agradecem ao CNPQ, à FINEP/PADCT e à FAPEMIG pelo apoio financeiro.

\section{REFERÊNCIAS}

[1] C. A. Baldwin, J. Coll. Int. Sci.181 (1996) 79.

[2] G. E. Mueler, Chem. Eng. Sci. 46, 2 (1991) 706.

[3] F. N. Rhines, R. T. DeHoff, J. Kronsbein, A topological study of the sintering process. U. S. Atomic Energy Commision, Gainesville, Florida, 1969.

[4] H. Martin, Chem. Eng. Sci. 33, 2 (1978) 913.

[5] J. Zheng, P. F. Johnson, Computer simulation of particle packing and sintering. Ph.D. Thesis, Alfred University, Alfred, NY, 1991.

[6] M. M. Schvartzman, V. Vasconcelos, Anais do $1^{\circ}$ Encontro Brasileiro Sobre Adsorção, Fortaleza, CE, 3 a 6 de Julho de 1996. (Rec. 4/97, Ac. 11/97) 